\title{
A Novel Topical Spray Formulation for Ginkgo Biloba for Antifungal Activity
}

\author{
Ahmed A. H. Abdellatif ${ }^{* *}$ and Heba A. Abou-Taleb²
}

${ }^{1}$ Department of Pharmaceutics and Industrial pharmacy, Faculty of Pharmacy, Al-Azhar University, Assuit 71524, Egypt ${ }^{2}$ Department of Pharmaceutics and Industrial pharmacy, Faculty of Pharmacy, Nahda University, Benisuef, Egypt

\begin{abstract}
The aim of this study is to prove the antifungal and antibacterial activity of Ginko biloba GKB from glycerin nanocapsules and nanoemulsions. GKB was formulated as spray formulation for topical application using ethyl alcohol $95 \% / g l y c e r o l / w a t e r$ based formulations. GKB spray was evaluated for their particle size distribution, $\mathrm{pH}$, content uniformity, content per spray, in vitro release and antifungal activity. The antifungal activity was determined by Agar welldiffusion method. The formulae were tested for their skin permeation and the stability of the formulations. The results showed uniform formulae with nano-sized particles, $\mathrm{pH}$ compatible with the topical formulation and higher drug content. Diffusion studies of the optimized formulations through the abdominal rabbit skin showed higher penetrated drug over a period of $24 \mathrm{~h}$. Stability studies indicated that formulations were stable. Skin irritation studies confirmed the compatibility of spray formulae with skin. Agar well-diffusion method showed that GKB was effective compared with fluconazole. The inhibition zones for all formulae were significant as compared with standard fluconazole. The results obtained showed that the topical spray formulation could be a promising and innovative therapeutic system as topical antifungal for the transdermal administration of GKB. The developed formulation can be used for delivery from spray formulation. This study proved the local activity of GKB as antifungal drugs.
\end{abstract}

Keywords: Topical spray; In vitro release; Ginkgo biloba

\section{Introduction}

Several different antifungal preparations were used to treat various fungal infections $[1,2]$. They are formulated as sprays, creams, solutions, ointment, shampoos, vaginal tablets, oral medicines, and injections [3]. The duration of treatment depends on kind of fungal infection, health problems of the infected patient, problems associated with immune system and types of formulations. The courses of treatment can be as short for few days or long for months [4].

Antifungal liquids or sprays (topical antifungals) can be used to treat skin fungal infections, scalp and nails [5]. These antifungal drugs can cause various type of adverse effect such as burning or redness at the site of application [6,7]. It was reported that 80 endophytic fungi were isolated from GKB, they were active against the chosen fungi using the agar diffusion method. The study indicated that GKB could be used as a fungicide to defend host plants against pathogenic organisms [8]. GKB has antifungal activity against some kind of endophytic. GKB extract holds flavonoid glycosides and unique terpene lactones as main active constituents [9].

Here GKB is presented for the $1^{\text {st }}$ time as a new finding antifungal drug for human. It was formulated as novel spray formulations as safe natural product which have no side effects and are easy to use. GKB spray is a favorite antifungal medicine for curing all types of fungal infections, since it can be used it to reduce itching, burning and scaling accompanied with all kinds of Tinea infections. The aim of this study was to formulate spray formula containing GKB and to verify the antifungal and antibacterial action of GKB. GKB was formulated as spray formulation for topical application using ethyl alcohol, glycerol and water established formulations. GKB spray was assessed for numerous parameters such as particle size distribution, $\mathrm{pH}$, drug content per spray, content uniformity, in vitro release and antifungal activity. The established formulation was used for delivery from spray formulation. The antifungal activity was determined by Agar welldiffusion method.

\section{Materials and Methods}

\section{Materials}

Ginkgo biloba was kindly supplied from Liptis pharmaceuticals, Cairo, Egypt. Glycerol, ethyl alcohol (95\%) and whatmann paper were purchased from, El-Gomhoria Chem. Co., Cairo (Egypt). Glass botle and spray pumps were purchased from Measom Freer, Wigston, UK. All chemicals were of analytical grades. All glasswares were thoroughly washed with freshly prepared aqua regia $\left(\mathrm{HCl}: \mathrm{HNO}_{3}, 3: 1\right)$, extensively rinsed with Millipore water several times and oven-dried at $50^{\circ} \mathrm{C}$ for 2-3 hours before use.

\section{Methods}

Selection of excipients: Formulae were prepared using aqueous, non-aqueous diluents, co-solvents, and permeation enhancers as shown in Table 1. Ethyl alcohol 95\% and water were selected as solvents for formulating of spray formulae due to the good solubility of GKB in these solvents [10]. Glycerol was used as a permeation enhancer and as coating material for GKB [11]. $F_{3}$ were found to be optimized formulation (Table 1).

Selection of containers and spray pumps: Glass bottles with a cubic figure carved at the base were employed for filling the transdermal spray formulae. The selected spray pumps can be used not only for body spray but also for hairspray, sunscreen, insect repellent, mist sprays, lens \& glass cleaning solutions [12]. Spray pumps were chosen with

*Corresponding authors: Ahmed AH Abdellatif, Department of Pharmaceutics and Industrial pharmacy, Faculty of Pharmacy, Al-Azhar University, Assuit 71524, Egypt, Tel: +201016660069; E-mail: ahmed.a.h.abdellatif@azhar.edu.eg

Received May 23, 2016; Accepted June 22, 2016; Published June 29, 2016

Citation: Ahmed A. H. Abdellatif, Heba A. Abou-Taleb (2016) A Novel Topical Spray Formulation for Ginkgo Biloba for Antifungal Activity. J Nanomed Nanotechnol 7: 386. doi:10.4172/2157-7439.1000386

Copyright: (c) 2016 Ahmed A. H. Abdellatif, et al. This is an open-access article distributed under the terms of the Creative Commons Attribution License, which permits unrestricted use, distribution, and reproduction in any medium, provided the original author and source are credited. 


\begin{tabular}{|c|c|c|c|}
\hline Parameter & F3 & $\mathbf{F 2}$ & F1 \\
\hline Spray pattern & $\begin{array}{l}\text { Have high uniformity and spreadability of } \\
\text { films. }\end{array}$ & $\begin{array}{l}\text { High precipitate and low uniformity of } \\
\text { films. }\end{array}$ & $\begin{array}{l}\text { High precipitate and low solubility of } \\
\text { films. }\end{array}$ \\
\hline Mean particle size $(\mathrm{nm})( \pm S D)$ & $555.6 \pm 2.5$ & $908.3 \pm 3.1$ & $901.1 \pm 1.2$ \\
\hline Evaporation time & $1-1.1 \mathrm{~m}$ & $1.2-1.3 \mathrm{~m}$ & $1.2-1.4 \mathrm{~m}$ \\
\hline Leakage from container & No leakage & No leakage & No leakage \\
\hline $\mathrm{pH}$ & 6-6.5 & $6.6-6.9$ & 7.1-7.2 \\
\hline Drug content per spray (\%) ( \pm SD) & $104.06( \pm 1.721)$ & $105.01( \pm 0.13)$ & $102.78( \pm 1.153)$ \\
\hline Content uniformity $(\%)( \pm S D)$ & $101.65( \pm 1.569)$ & $103.78( \pm 0.462)$ & $104.02( \pm 1.432)$ \\
\hline Poly dispersity Index (PDI) & 0.3 & 0.4 & 0.33 \\
\hline Zeta potential & $+43.7 \pm 3.23$ & $+33.7 \pm 2.9$ & $+56 \pm 11$ \\
\hline
\end{tabular}

Table 1: Characterization of ginko biloba spray formulations.

over-cap (Diameter $18 \mathrm{~mm}$, Height $39 \mathrm{~mm}$, Neck Size $15 \mathrm{mmR}$, Dose $120 \mu \mathrm{l}$, min 1,000 for Spray Pump) as it is more suitable for body spray.

Formulation development of topical spray preparation: GKB spray formulations were developed as topical solutions made up of ethyl alcohol 95\%, and water dissolved in one phase [10]. Glycerol was used in this formula as enhancer and as coating material for GKB [11]. Briefly, GKB was dissolved in required amount of water and stirred for $1 \mathrm{hr}$ as. The required amount of glycerol were added to the aqueous solution of GKB and stirred for another $1 \mathrm{hr}$. The dissolved GKB solution were dropped on the half amount of ethyl alcohol $95 \%$ and stirred for $3 \mathrm{hrs}$. The total volume of the system was adjusted with ethyl alcohol $95 \%$ to $1 \mathrm{~L}$. GKB were formulated as spray using ethyl alcohol $95 \%$, water and glycerol in different concentrations. GKB was dissolved in this aqueous solution with concentration of $6 \mathrm{gm} / 1 \mathrm{~L}$. The overall solution containing GKB was stirred for $1 \mathrm{hr}$ to completely dissolve GKB. GKB solution was purified using whatmann paper. GKB solution was accurately filled in specially designed glass bottles capped with atomizer.

\section{Characterization of developed spray formulations}

Particle size distributions: Particle size distributions were achieved using the Malvern zetasizer nano 6.01 (Malvern Instruments Ltd, UK). The sampling time was set automatically; three measurements were permitted to perform each 10 sub-runs. All measurements were carried out in alcoholic solution [13-17].

Evaporation time and pH of solution: To test the evaporation time, GKB formulae were sprayed on ethyl alcohol sensitive paper and the drying time or evaporation time were noted. The $\mathrm{pH}$ value of the spray holding GKB was estimated using a digital $\mathrm{pH}$ meter $(3500 \mathrm{pH}$ meter, Jenway, UK). The transparency and thickness of the misted films were also reported $[18,19]$

Efficiency of the pump seal: Efficiency of the pump seal and its ability to stock the contents of the product was estimated by leak test/ Pump seal effectiveness test. The occupied containers under test were located in the upright position at $30^{\circ}$ for known time. The bottles were balanced before and after the test stage. The variation in the weight of the bottle was written down and the leakage rates were anticipated [18-20].

Drug content: The drug content per spray was determined spectrophotometerically at $\lambda_{\max } \quad 380 \mathrm{~nm}$ using UV-Visible Spectrophotometer (Shimadzu, Japan, UVPC personal spectroscopy, software version 2), and the drug content was determined using equation 1 . Briefly, $1 \mathrm{~mL}$ of this solution was diluted with citrate buffer to $25 \mathrm{~mL}$ in volumetric flask. Then the solution was analyzed for GKB conc spectrophotometrically at $\lambda_{\max } 380 \mathrm{~nm}$ against a blank solution prepared in the same manner using aqueous alcoholic spray [19-22].

Drug content $\%=[$ Concentration of entrapped drug] $/[$ Total drug concentration] $\times 100(1)$

In vitro skin permeation experiments: In vitro penetration studies were carried out using abdominal male rat skin $(150 \pm 25 \mathrm{~g})$. To obtain the skin of the rats, animals were first sacrificed. It was fixed on a modified Franz diffusion cell with a surface area of $7.0 \mathrm{~cm}^{2}$. The dissolution medium was citrate buffer at $\mathrm{pH} 6$ thermostatic at $37^{\circ}$ and stirred with a magnetic stirrer. One $\mathrm{mL}$ of this solution was diluted with citrate buffer to $25 \mathrm{~mL}$ in volumetric flask. Then the solution was analyzed for GKB spectrophotometerically at $\lambda_{\max } 380 \mathrm{~nm}$ against a blank solution prepared in the same manner using GKB spray $[18,19,21,22]$. Each penetration experiment was frequented 6 times. Percentage cumulative amounts of drug penetrated were plotted as a function of time. Approval of the Institutional Animal Ethical Committee was obtained prior to the commencement of skin irritation studies from the faculty of medicine, Asyut University, Asyut, Egypt. Moreover, the flux was calculated from the slope of the linear portion of the obtained curve. The permeability coefficient $\left(K_{p}\right)$ of GKB crossways rat skin was calculated using Fick's first law of diffusion, which is expressed by the following equation:

$$
K p=\frac{J}{C}(2)
$$

Where; $J$ is the flux $\left(\mathrm{mg} / \mathrm{cm}^{2} / \mathrm{hr}\right)$ and $\mathrm{C}$ is the drug concentration in donor compartment.

Stability studies: The conventional formulations were set aside for stability testing. The chemical stability of the formulations was estimated by valuation of the percentage drug residual in the formulations, drug release pattern, observing any change in $\mathrm{pH}$, appearance, spray pattern, leakage rate and average weight per actuation assessed physical stability $[14,18]$.

\section{Skin irritation studies}

The optimized formulations were sprayed on the scrap of shaved skin and observing the resulting reactions such as erythema and edema after $24 \mathrm{~h}$. Briefly, five human volunteers were selected with age of 30-40 years, having no history of allergy against GKB, and the test formulation was applied to the back of hand on an area of 2 square inch. Then, the volunteers were observed for appearance of lesions or irritation $[14,18]$

Antifungal activities: The study of antifungal activity for GKB spray formula was carried out using different strains of fungi and bacteria. The antifungal activity of dry powder of GKB formulae was determined 
by agar well-diffusion method $[23,24]$. The media used was MuellerHinton agar at only $4 \mathrm{~mm}$ deep, poured into $100 \mathrm{~mm}$ or $150 \mathrm{~mm}$ Petri dishes. The $\mathrm{pH}$ level of the agar must be between 7.2 and 7.4. Briefly, the work was done using aseptic technique. A sterile swab was placed into the broth culture of a specific organism and then the excess liquid was removed by gently pressing or rotating the swab against the inside of the tube. To obtain uniform growth, the plate was streaked with the swab in one direction, the plate was rotated at $90^{\circ}$ and the plate was splashed again in that direction. The plate was allowed to dry for approximately 5 minutes. Antibiotic discs containing specific antibiotics were distributed onto the plate. Using a flame-sterilized forceps, each disc was gently pressed to the agar to ensure that the disc is attached to the agar. The plates were incubated overnight at an incubation temperature of $37^{\circ} \mathrm{C}\left(98.6^{\circ} \mathrm{F}\right)$. Inoculation was made with a diluted broth culture to match a $0.5 \mathrm{McF}$ arland turbidity standard, which is roughly equivalent to 150 million cells per $\mathrm{mL}$. The inhibition zones for all GKB formulae were measured as compared with fluconazole as a standard antifungal.

Statistical analysis: All statistical analysis was performed using One-way analysis of variance ANOVA. Minitab 16 Statistical Software with the Tukey's multiple comparison was employed for comparing the formulations with each other. Statistically significant differences were assumed when $\mathrm{p}<0.05$. All values are expressed as their mean \pm standard deviation [18].

\section{Result and Discussion}

\section{Spray formulae}

Formula consisting of ethyl alcohol 95\%: glycerol: water in the ratio 7:2:1 exhibited preferred spray patterns with high and good dispersibility of the GKB F3. The optimized formulae were selected based on the transparency of the solution, dehydration rate, spray display and thickness of the film formed. Formulation F1 was a simple drug solution in solvent system and showed high clarity as it has a high concentration of ethyl alcohol but the film was thin and was not stable. The films formed by F3 were transparent and cohesive. The organic solvents in the formulations vaporized rapidly leaving behind a thin film that adhered to the skin for periods up to $4 \mathrm{~h}$. Comparing the physicochemical characteristics of the films, spray patterns and drug content, formulations F1, F2 and F3. F3 were found to be better as compared to other formulations. The detailed results of qualitative and quantitative tests for reference formulation and the optimized formulations (F2 and F3) are summarized.

Size, zeta potential and PDI are factors that designate the stability of the obtained nanoparticles [25-28]. The surface charge plays an essential role in the stability of the obtained nanoparticles and the degree of zeta potential is indicative of the colloidal stability of the system [29]. The results showed a satisfactory small and uniform particle size. The formulated nanoparticles were of a uniform $\mathrm{Z}$ average diameter and nano-size range, Figure 1. DLS recorded only one peak with intensity

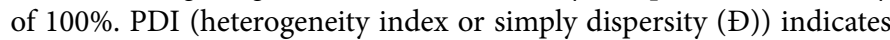
the stability of the product since it denotes the particles distribution in colloidal solution. Smaller PDI suggest the homogeneity of the particle size in suspension, ideally the value of PDI should be $<0.70$ because they indicated a particle size distribution falls within a narrow range of sizes [27,30-33]. In the present study all PDI were of acceptable range as recorded by DLS for all GKB spray, indicating the homogeneity of the particle size in the obtained suspension as showed in Figure 1.

The zeta potential zeta potential of all particle types was positive, Figure 2 . GKB had a highly positively zeta potential, indicating that GKB was coated with repulsive force enough to be stable. DLS recorded also high-count rate $(185 \mathrm{kpcs})$ indicates that the concentration of nanoparticles was high enough for measurements.

The spray display was found to be affected by the size and shape of actuator orifice as well as by the capacity of pump. Formula F3 showed good spray displays in terms of uniform and spherical messes due to the supple and cohesive film of the glycerol used.

F3 showed $\mathrm{pH}$ in the range of 6.0-6.5 which mimicking the skin $\mathrm{pH}$ The $100 \mu \mathrm{l}$ pumps delivered average weight of $70 \pm 5.0 \mathrm{mg}$ per actuation

\begin{tabular}{|c|c|c|}
\hline Formulation & Flux $\left(\mathbf{m g} / \mathbf{c m}^{\mathbf{2}} \mathbf{. h}\right)$ & \\
\hline F1 & 0.0177 & ${ }^{*} \mathbf{Q}_{\mathbf{2 4}}\left(\mathbf{m g} / \mathbf{c m}^{\mathbf{2}}\right)$ \\
\hline F2 & 0.4374 \\
\hline F3 & 0.0206 & 0.4663 \\
\hline
\end{tabular}

*Amount of drug released per unit surface area after $24 \mathrm{~h}$. Formulations $\mathrm{F} 1$ and F3 showed zero order kinetic model while formulation F2 showed root time kinetics with $\mathrm{R}^{2}$ value of 0.9608 . [Formulations $\mathrm{F} 2$ and F3 (containing film formers and penetration enhancers) showed more flux and $\mathrm{Q}_{24}$ value for the drug as compared to Formulation F1]

Table 2: Permeation parameters of ginko biloba across rabbit abdominal skin.

Size Distribution by Intensity

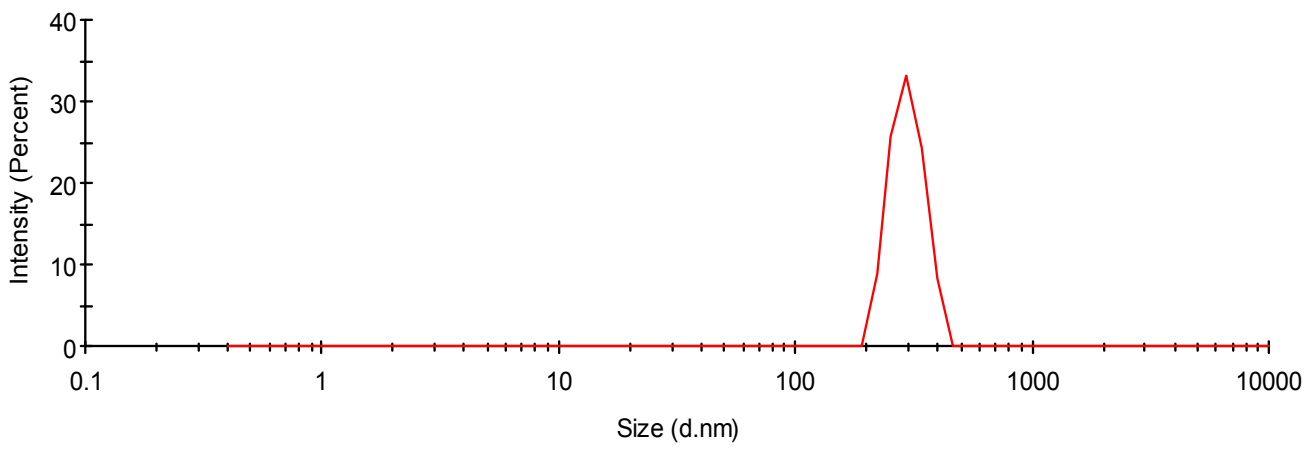

Figure 1: Size distribution of ginko biloba solution for formula F3 as determined by zetasizer nano. 
$(n=5)$. There was no statistically significant variation in the amount emitted per actuation indicating the effectiveness of the pump system in delivering reproducible amounts of the formulation per actuation (Table 1). Formulations F1, F2 and F3 showed average drug contents per spray of 100 to $104 \%$. This test indicates the amount of the therapeutically active ingredient delivered per metered spray from the metered dose containers. Formulation F3 was found to show better uniformity in terms of content per spray. We inferred that the formulation F3 had more uniformity of the dosage of the active ingredient delivered through the metered pump compared to formulations F1 and F2.

\section{Ex vivo skin permeation}

Permeation properties of the GKB were illustrated initially by conducting diffusion studies on the simple organic drug solution (F3). The diffusion studies from the optimized formulations F1, F2 and F3 were carried out over a period of $24 \mathrm{~h}$. Rabbit abdominal skin was used as animal model skin, because of its similarity with human skin ex vivo. As illustrated in Figure 3, a linear correlation was achieved between $\mathrm{Q}$ (the amount cumulative of penetrated GKB across the unit surface area of the skin) and time up to $24 \mathrm{~h}$. The formulation F3 exhibited significantly higher penetration $(\mathrm{P} \leq 0.05)$ of $\mathrm{GKB}$ as compared to formulation F1 and F2. The reason for this higher permeation is due to the presence of high concentration of glycerol. Formula F3 contained glycerol with high concentration as the permeation enhancers leading to more permeation of drug into the skin. The same results were confirmed the penetration enhancer of glycerol were presented previously $[20,34,35]$.

\section{Statistical analysis}

The results illustrated in Table 2 confirmed the release kinetics fits the zero order kinetic models for all formulations. Analysis of variance showed that these correlations are statistically significant $(P \leq 0.05)$ Formula F3 holding higher concentration of glycerol as penetration enhancer showed more flux and $\mathrm{Q}_{24}$ value for the drug as compared to the other formulations F1 and F2.

\section{Stability studies}

Stability studies showed the formulations to be stable in terms of physicochemical parameters, drug content and drug release, under all the storage conditions for F 3 as selected to be the best formulation as shown in Table 3

\section{Skin irritation studies}

No erythema or edemas were observed in the primary skin

Zeta Potential Distribution

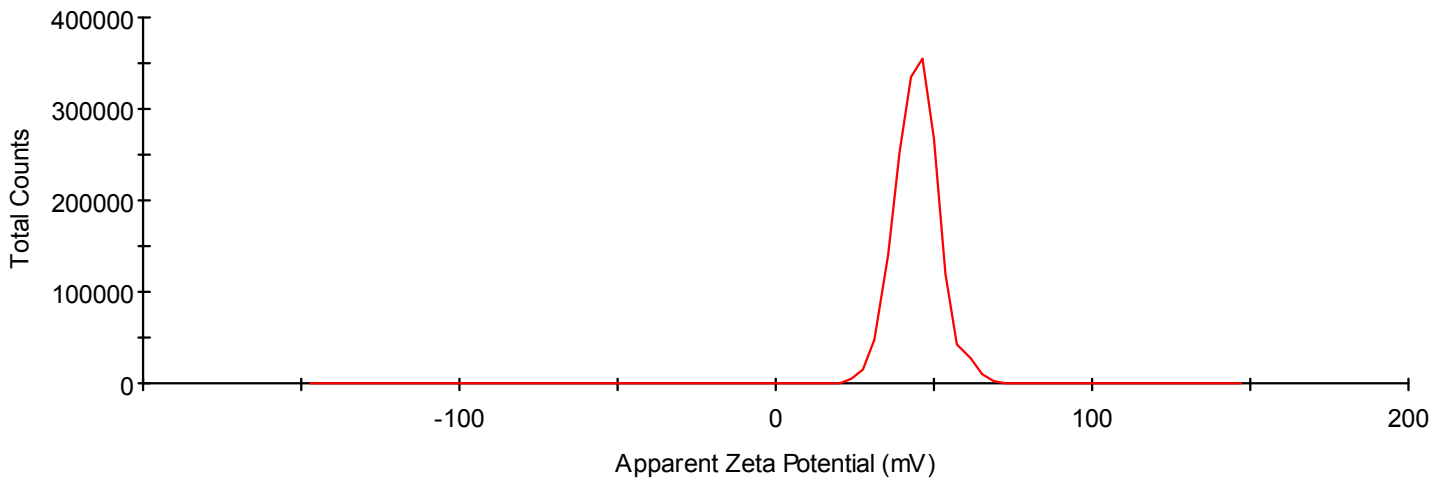

Figure 2: Zeta potential measured using zetasizer nano for formula F1.

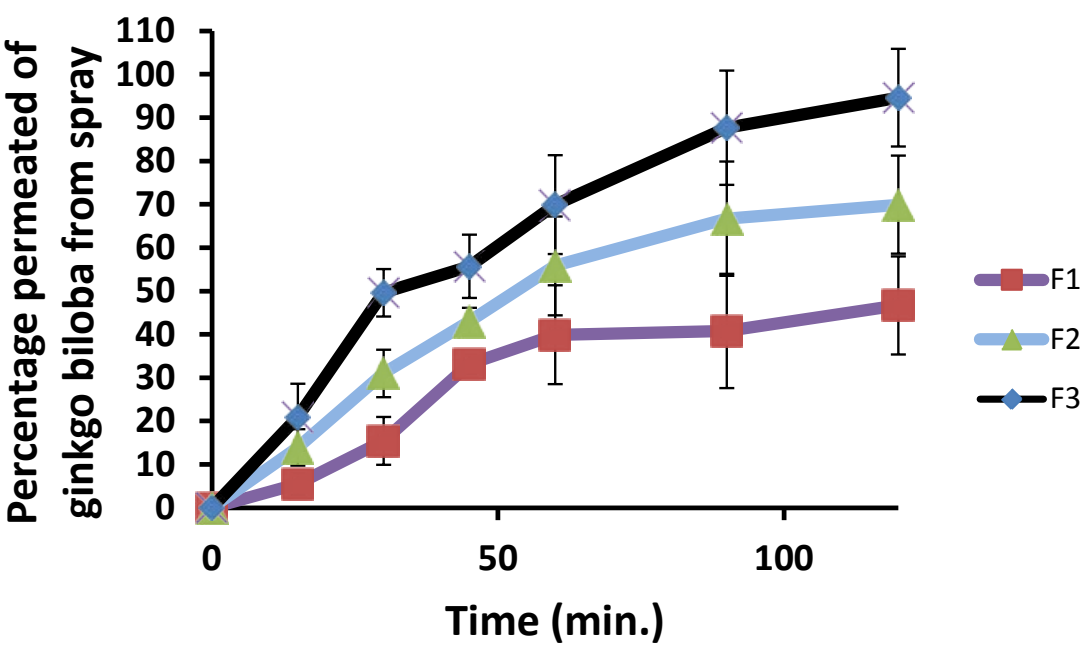

Figure 3: Percentage permeation of ginkgo biloba from spray solution across skin rabbit. 
Citation: Ahmed A. H. Abdellatif, Heba A. Abou-Taleb (2016) A Novel Topical Spray Formulation for Ginkgo Biloba for Antifungal Activity. J Nanomed Nanotechnol 7: 386. doi:10.4172/2157-7439.1000386

Page 5 of 6

\begin{tabular}{|c|c|c|c|}
\hline \multicolumn{4}{|c|}{ Stability temperature at $37 \pm 2^{\circ}$ for $F 1$} \\
\hline Parameters evaluated & Freshly prepared & After 1 month & After 3 months \\
\hline Appearance & Clear & Clear & Clear \\
\hline $\mathrm{pH}( \pm \mathrm{SD})$ & $6.2 \pm 0.05$ & $6.4 \pm 0.01$ & $6.5 \pm 0.02$ \\
\hline Spray pattern & Uniform & Uniform & Uniform \\
\hline Percent drug remaining $( \pm S D)$ & $100.07 \pm 0.45$ & $99.75 \pm 1.31$ & $99.52 \pm 1.07$ \\
\hline
\end{tabular}

Table 3: Stability studies data of the optimized ginko biloba spray formulations at different temperature conditions, after three months $(n=3)$.

\begin{tabular}{|c|c|c|}
\hline \multirow{2}{*}{ Microorganisms } & \multicolumn{2}{|c|}{ Treatments } \\
\cline { 2 - 3 } & Fluconazole & GKB \\
\hline Bacillus subtilis & $16.0 \pm 0.8$ & $13.9 \pm 0.3$ \\
\hline Escherichia coli & $20.6 \pm 1.4$ & $18.4 \pm 0.5$ \\
\hline Candida albicans & $19.5 \pm 1.0$ & $13.3 \pm 1.3$ \\
\hline Candida glabrata & $25.4 \pm 1.7$ & $16.6 \pm 0.3$ \\
\hline Epidermophyton floccosum & $14.1 \pm 1.6$ & $10.2 \pm 1.2$ \\
\hline Trichophyton rubrum & $20.9 \pm 2.7$ & $12.1 \pm 1.6$ \\
\hline Trichophyton verrucosum & $21.3 \pm 1.4$ & $16.0 \pm 0.4$ \\
\hline
\end{tabular}

5. Carrillo-Munoz AJ, Tur-Tur C, Cardenes D, Rojas F, Giusiano G (2015) [In vitro antifungal susceptibility profile of Scopulariopsis brevicaulis isolated from onychomycosis]. (Translated from spa) Rev Esp Quimioter 28(4): 210-213 (in spa).

6. Kumar L, Verma S, Bhardwaj A, Vaidya S, Vaidya B (2014) Eradication of superficial fungal infections by conventional and novel approaches: a comprehensive review. (Translated from eng) Artif Cells Nanomed Biotechnol 42(1): $32-46$ (in eng)

7. Subissi A, Monti D, Togni G, Mailland F (2010) Ciclopirox: recent nonclinica and clinical data relevant to its use as a topical antimycotic agent. (Translated from eng) Drugs 70(16): 2133-2152 (in eng).

Table 4: Antibacterial and antifungal activity of ginko biloba using the well diffusion method (Inhibition zone in $\mathrm{mm}$ ).

irritation studies of the optimized formulations on the rabbits. It found to be safe and non-irritant for transdermal application. The formulation developed was more efficient for a period of $24 \mathrm{~h}$ after application. However results need to be confirmed by pharmacokinetic studies.

\section{Antifungal activities}

This study proved the local activity of GKB as antifungal drugs. Vertical distribution of microfungi on decomposing GKB powder is shown in Table 4, in which the calculated occurrence frequencies are also indicated. Seven species of microfungi were recorded from powder extract of GKB. As listed previously, antifungal activities have been recorded for GKB as compared with fluconazole itself as reference antifungal. All the recorded results suggested the antifungal activity of GKB even fluconazole is better.

\section{Conclusions}

The evidence suggests that GKB is more effective as local antifungal action in comparing to fluconazole. GKB was successfully formulated as solution for spray pattern which can be used in the future trials as topical formulae on numbers of participants. From the results obtained in the present work, it can be concluded that GKB can be an innovative and promising approach for the transdermal administration of GKB. The diffusion studies indicated that the permeation of GKB formulations through the skin was much higher as compared to the diffusion of simple organic solution of the drug.

\section{References}

1. Ginovyan M, Keryan A, Bazukyan I, Ghazaryan P, Trchounian A (2015) The large scale antibacterial, antifungal and anti-phage efficiency of Petamcin-A: new multicomponent preparation for skin diseases treatment. (Translated from eng) Ann Clin Microbiol Antimicrob 14: 28 (in eng).

2. Pagano $L$ (2013) Combined antifungal approach for the treatment of invasive mucormycosis in patients with hematologic diseases: a report from the SEIFEM and FUNGISCOPE registries. (Translated from eng) Haematologica 98(10): e127-130 (in eng).

3. Mathpal D, Garg T, Rath G, Goyal AK (2015) Development and characterization of spray dried microparticles for pulmonary delivery of antifungal drug. Curr Drug Deliv 12: 464-471.

4. Ribeiro RF (2016) Spray-dried powders improve the controlled release of antifungal tioconazole-loaded polymeric nanocapsules compared to with lyophilized products. (Translated from eng) Mater Sci Eng C Mater Biol Appl 59: 875-884 (in eng).

8. Xiao Y (2013) Antifungal screening of endophytic fungi from Ginkgo biloba for discovery of potent anti-phytopathogenic fungicides. (Translated from eng) FEMS Microbiol Lett 339(2): 130-136 (in eng).

9. Cao LL, Zhang YY, Liu YJ, Yang TT, Zhang JL, et al. (2016)Anti-phytopathogenic activity of sporothriolide, a metabolite from endophyte Nodulisporium sp. A21 in Ginkgo biloba. Pestic Biochem Physiol 129: 7-13.

10. Wei Y, Wang Y, Wang L, Hao D, Ma G (2011) Fabrication strategy for amphiphilic microcapsules with narrow size distribution by premix membrane emulsification. (Translated from eng) Colloids Surf B Biointerfaces 87(2): 399408 (in eng).

11. Emami J, Mohiti H, Hamishehkar H, Varshosaz J (2015) Formulation and optimization of solid lipid nanoparticle formulation for pulmonary delivery of budesonide using Taguchi and Box-Behnken design. Res Pharm Sci 10: 17-33.

12. Loretz L, Api AM, Barraj L, Burdick J, Davis de A, et al. (2006) Exposure data for personal care products: hairspray, spray perfume, liquid foundation, shampoo, body wash, and solid antiperspirant. Food Chem Toxicol 44: 2008-2018.

13. Abdellatif AA (2015) Targeting of Somatostatin Receptors using Quantum Dots Nanoparticles Decorated with Octreotide. Journal of Nanomedicine \& Nanotechnology 2015.

14. Abdellatif AA, Abou-Taleb HA (2015) Optimization of nano-emulsion formulations for certain emollient effect. WJPPS

15. Abdellatif AA, El Rasoul SA, Osman S (2015) Gold nanoparticles decorated with octreotide for somatostatin receptors targeting. Int J Pharm Sci Res 7(1): 14-20

16. Abdellatif AA, Zayed G, El-Bakry A, Zaky A, Saleem IY, et al. (2016) Nove gold nanoparticles coated with somatostatin as a potential delivery system for targeting somatostatin receptors. Drug Dev Ind Pharm .

17. Ahmed AH, Abdellatif HAA-T (2016) Transfersomal Nanoparticles of Keratolytic and Antibacterial Agents for Enhanced Transdermal Delivery. Journal of Nanotechnology \& Advanced Materials 4(1): 19-23.

18. Abdellatif AA, Tawfeek HM (2015) Transfersomal Nanoparticles for Enhanced Transdermal Delivery of Clindamycin. (Translated from Eng) AAPS PharmSciTech (in Eng).

19. Abdellatif AAH, El Hamd MA, Saleh KI (2016) A Formulation, Optimization and Evaluation of Controlled Released Alginate Beads Loaded-Flurbiprofen. $J$ Nanomed Nanotechnol 7(357): 2.

20. Bakshi A, Bajaj A, Malhotra G, Madan M, Amrutiya N (2008) A novel metered dose transdermal spray formulation for oxybutynin. Indian J Pharm Sci 70: 733739 .

21. Akhlaq M (2011) A simple high-performance liquid chromatographic practica approach for determination of flurbiprofen. (Translated from eng) J Adv Pharm Technol Res 2(3): 151-155 (in eng)

22. El Hamd MA, Abdellatif AAH, Derayea SM, Abdelmageed OH, Askal HF (2015) Spectrophotometric Determination of Nifedipine and Nicardipine in their Pharmaceutical Preparations. Industrial Chemistry 2015. 
Citation: Ahmed A. H. Abdellatif, Heba A. Abou-Taleb (2016) A Novel Topical Spray Formulation for Ginkgo Biloba for Antifungal Activity. J Nanomed Nanotechnol 7: 386. doi:10.4172/2157-7439.1000386

23. Athanassiadis B, Abbott PV, George N, Walsh LJ (2009) An in vitro study of the antimicrobial activity of some endodontic medicaments and their bases using an agar well diffusion assay. (Translated from eng) Aust Dent J 54(2): 141-146 (in eng).

24. Holder IA, Boyce ST (1994) Agar well diffusion assay testing of bacterial susceptibility to various antimicrobials in concentrations non-toxic for human cells in culture. (Translated from eng) Burns 20(5): 426-429 (in eng).

25. Abdellatif AA (2015) Targeting of somatostatin receptors using quantum dots nanoparticles decorated with octreotide. Journal of Nanomedicine \& Nanotechnology S6: 1-8.

26. Abdellatif AA, Tawfeek HM (2015) Transfersomal nanoparticles for enhanced transdermal delivery of clindamycin. AAPS PharmSciTech 28: 28.

27. Pereira-Lachataignerais $\mathrm{J}$, Pons $R$, Panizza $P$, Courbin $L$, Rouch $\mathrm{J}$, et al. (2006) Study and formation of vesicle systems with low polydispersity index by ultrasound method. Chem Phys Lipids 140: 88-97.

28. Moraes C, De Paula E, Rosa A, Fraceto L (2010) Physicochemical stability of poly(lactide-co-glycolide) nanocapsules containing the local anesthetic bupivacaine. Journal of the Brazilian Chemical Society 21(6): 995-1000.

29. Kaufman E (2007) Probing protein adsorption onto mercaptoundecanoic acid stabilized gold nanoparticles and surfaces by quartz crystal microbalance and zeta-potential measurements. Langmuir 22(23): 6053-6062.

30. Aghajani M, Shahverdi AR, Amani A (2012) The use of artificial neural networks for optimizing polydispersity index (PDI) in nanoprecipitation process of acetaminophen in microfluidic devices. AAPS PharmSciTech 13: 1293-1301.

31. Lewis G, Li Y (2010) Dependence of in vitro fatigue properties of PMMA bone cement on the polydispersity index of its powder. J Mech Behav Biomed Mater 3: $94-101$

32. Rosenfeld C, Serra C, Brochon C, Hadziioannou G (2008) Influence of micromixer characteristics on polydispersity index of block copolymers synthesized in continuous flow microreactors. Lab Chip 8: 1682-1687.

33. Viéville J, Tanty M, Delsuc MA (2011) Polydispersity index of polymers revealed by DOSY NMR. J Magn Reson 212: 169-173.

34. Lin CH, Fang CL, Al-Suwayeh SA, Yang SY, Fang JY (2011) In vitro and in vivo percutaneous absorption of seleno-L-methionine, an antioxidant agent, and other selenium species. Acta Pharmacol Sin 32: 1181-1190.

35. Abdelrahman FE, Elsayed I, Gad MK, Badr A, Mohamed MI (2015) Investigating the cubosomal ability for transnasal brain targeting: In vitro optimization, ex vivo permeation and in vivo biodistribution. Int J Pharm 490: 281-291. 